BASIC RESEARCH

\section{Visceral adipose tissue regulates cardiac ageing}

Osteopontin (OPN) is a matricellular protein that is upregulated in models of pathological cardiac fibrosis and remodelling. OPN plasma levels also increase with age, and a new study published in Circulation reveals that OPN secreted by visceral adipose tissue (VAT) promotes age-associated interstitial fibrosis in the heart.

Ageing induces structural changes in the heart, notably fibrotic remodelling, which impairs cardiac function. Several matricellular proteins have previously been implicated in fibrotic cardiomyopathy in ageing individuals but the role and source of OPN during cardiac ageing are unknown. Sawaki and colleagues analysed OPN expression in tissues from young and aged mice and showed that VAT is the main source of OPN during ageing, whereas the myocardium expresses a low, stable level of OPN. Surgical removal of VAT in aged mice reduced levels of OPN and the profibrotic cytokine transforming growth factor- $\beta 1$ in the circulation, decreased cardiac fibrosis, and improved myocardial function. Knocking out $O p n$ abolished the increase in myocardial interstitial fibrosis and the reduction in myocardial contractility associated with ageing.
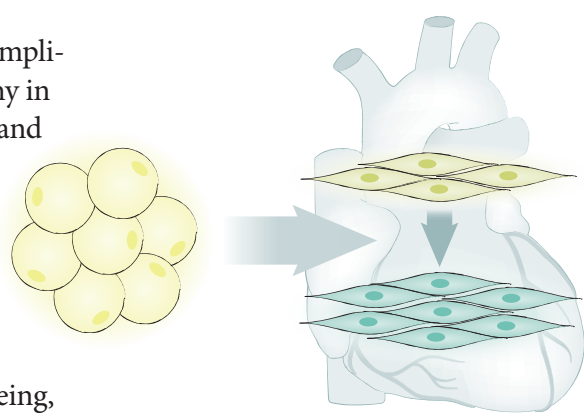

Primary fibroblasts isolated from newborn mice were then incubated with plasma from wild-type or $\mathrm{Opn}^{-/}$ aged mice, which revealed that depletion of OPN in the plasma reduced the activation and proliferation of fibroblasts while promoting cellular senescence. Together, these results demonstrate that VAT drives cardiac ageing by releasing profibrotic factors such as OPN, which promote cardiac fibroblast activation over senescence.

Pharmacological inhibition of OPN in aged mice reduced OPN expression in VAT, improved agedependent cardiac remodelling and function, and increased senescence. Therefore, new therapies targeting OPN should be investigated to prevent the development of myocardial interstitial fibrosis and to promote healthy cardiac ageing.

Alexandra Le Bras

ORIGINAL ARTICLE Sawaki, D. et al. Visceral

adipose tissue drives cardiac aging through modulation of fibroblast senescence by osteopontin

production. Circulation https://doi.org/10.1161/

CIRCULATIONAHA.117.031358 (2018)

FURTHER READING Hassan, M. et al. Adipose

tissue: friend or foe? Nat. Rev. Cardiol. 9, 689-702 (2012) 